\title{
Biomechanics of the elbow joint in tennis players and relation to pathology
}

\author{
Denise Eygendaal, F T G Rahussen, R L Diercks
}

Br J Sports Med 2007;41:820-823. doi: 10.1136/bjsm.2007.038307

Elbow injuries constitute a sizeable percentage of tennis injuries. A basic understanding of biomechanics of tennis and analysis of the forces, loads and motions of the elbow during tennis will improve the understanding of the pathophysiology of these injuries. All different strokes in tennis have a different repetitive biomechanical nature that can result in tennis-related injuries. In this article, a biomechanically-based evaluation of tennis strokes is presented. This overview includes all tennisrelated pathologies of the elbow joint, whereby the possible relation of biomechanics to pathology is analysed, followed by treatment recommendations.

See end of article for authors' affiliations

Correspondence to: Denise Eygendaal, Amphia Hospital, Teteringen, The Netherlands; denise@ eygendaal.nl

Accepted 26 June 2007

Published Online First

17 July 2007
$\mathrm{T}$ he increase in the number of participants in sports as baseball, tennis, American football and volleyball has resulted in a sharp rise of sports injuries and thus to an increased incidence of elbow injuries in recent decades.

In the Netherlands, a study was conducted amongst all patients that received treatment at the emergency ward of general hospitals between 1998-2001; the injury risk was calculated for different group of patients in relation to type of racquet sport, age and sex.

In tennis the injury risk was fairly similar for males and females; the older age groups were affected more often and indoor tennis was related to a higher incidence of injuries in comparison to outdoor tennis.

The magnitude of forces across the elbow during tennis strokes can produce tremendous valgus and extension overload in players. The game of tennis has been described as a power game because of the high ball velocities and the explosive physical action of the players. ${ }^{2}$ Biomechanical analysis of these forces, loads and motion on the elbow in tennis will lead to an improved understanding of the pathophysiology of injuries in tennis.

Common injuries encountered include ulnar collateral ligament (UCL) tears, flexor-pronator muscle tendinosis or tears, ulnar neuritis, posterior impingement, ostechondritis dissecans of the capitellum and tendinopathy of the extensors, whereby the tendinopathy probably has the highest prevalence in tennis players.

The purpose of this article is to give an overview of elbow injuries and their treatment in adult tennis players in relation to the current knowledge regarding biomechanics of the elbow in tennis.
BIOMECHANICS OF THE ELBOW IN TENNIS

In the normal elbow joint, stability is maintained by the combination of joint congruity, capsuloligamentous integrity and well balanced intact muscles. The olecranon and olecranon fossa joint provide primary stability at less than $20^{\circ}$ or more than $120^{\circ}$ of elbow flexion. In between stability is provided by soft tissue constraints, mainly the UCL. $^{3} 4$

The kinetic chain of the tennis service starts with the feet and knees and travels through legs, trunk/back and shoulder to the elbow joint and finally through the wrist and hand. Biomechanically, the elbow functions primarily as a link in this kinetic chain, allowing transfer of kinetic energy from the body to the racquet.

High-speed video analysis studies from Kibler et $a l^{5}$ have demonstrated that during the service the elbow moves from $116^{\circ}$ to $20^{\circ}$ of flexion within $0.21 \mathrm{~s}$, with ball impact occurring at approximately $35^{\circ}$ of flexion. During groundstrokes, observed flexion and extension range was much less averaging $11(46-35)^{\circ}$ of flexion on the forehand and $18(48-30)^{\circ}$ in the backhand. The calculated angular velocity during the service motion was $982 \%$ for elbow extension. ${ }^{5}$

These data reveal the extreme forces that the elbow must repetitiously absorb during tennis strokes in flexion and extension direction.

In most sports with overhead movement, including tennis, an increased external rotation of the shoulder develops at the cost of internal rotation. Elliot has stressed the important role of internal rotation of the upper arm at the shoulder during service and forehand strokes. ${ }^{6}$ This demand on internal rotation of the upper arm during tennis can result in an increase of internal rotatory forces across the elbow joint.

In the third plane, valgus and varus, we also can observe abnormal load transfer in tennis. During normal elbow motion the axis of the elbow is from varus into valgus as it moves from flexion to extension. ${ }^{3}$

This combination of valgus forces and rapid extension during tennis results in tensile forces along the medial side, compression on the lateral portion of the elbow and shear forces in the posterior compartment. This combination is often called "valgus extension overload" syndrome in overhead athletes and can play a role in some injuries in the elbow in tennis players. $^{78}$ In

Abbreviations: $A O L$, anterior oblique ligament; $O D$, osteochondritis dissecans; POL, posterior oblique ligament; $\mathrm{UCL}$, ulnar collateral ligament 
summary, the tennis stroke puts very high loads on the elbow joint in extension, internal rotation and valgus, and this occurs in repetitive movements at very high speeds with very high forces.

\section{INSUFFICIENCY OF UCL}

The UCL complex consists of an anterior oblique ligament (AOL), posterior oblique ligament (POL) and a transverse band. The AOL has been shown to be the most important soft-tissue constraint to valgus instability of the elbow and is the strongest and stiffest of the collateral ligaments of the elbow with an average failure load of $260 \mathrm{~N}$. The AOL is also the primary stabiliser to internal rotatory forces. ${ }^{3}{ }^{4}$

The flexor carpi ulnaris muscle, pronator teres and flexor digitorum superficialis form predominantly the musculotendinous unit overlying the AOL; all three muscles have been described to contribute to medial support as secondary stabilisers.

Acute rupture and chronic overload of the UCL has been described extensively in athletes, especially in baseball pitchers. $^{8-12}$ Findings in acute medial collateral ligament injury are moderate to severe elbow pain, acute onset of pain during service or a popping sensation followed by medial ecchymosis or even acute ulnar nerve symptoms. Chronic overuse of the elbow, as described before, can also result in progressive attenuation of the UCL leading to ligamentous insufficiency even in the absence of a singular catastrophic episode of ligament failure. At physical examination the instability can be revealed; the degree of laxity is often underestimated. In patients with insufficiency of the UCL a typical painful arc can be produced using the "milking manoeuvre". ${ }^{12}$ The diagnosis is confirmed by positive elbow MRI, dynamic stress radiographs, dynamic ultrasonography or positive valgus test at anaesthesia. Treatment can be conservative or surgical. ${ }^{13}$

The previous described valgus and internal rotatory forces result in microtrauma of the UCL and eventually attenuation of the ligament. Attenuation of UCL leads to abnormal valgus movement of the elbow joint affecting the mechanics of the highly constrained articulation of the posterior elbow. This results in bony impingement at the superomedial corner or the olecranon and the corresponding fossa. Such impingement can lead to chondral lesion and eventually reactive changes such as osteophytic spur formation. ${ }^{14}{ }^{15}$

In prevention and treatment of UCL insufficiency in tennis players, therapy should be based on the above-mentioned biomechanics overload in all three planes of movement, extension, rotation and valgus. The "axis of internal rotation" of the humerus should be addressed, with optimal internal rotation of the shoulder, in combination with a proper technique of groundstrokes and service in which the extension should be monitored carefully. The flexor carp ulnaris muscle, pronator terse and flexor digit rum superficialis have been described to contribute to medial stability as secondary stabilisers. Specific training should be structured to these muscles to enhance valgus stability of the elbow joint. ${ }^{16}$

\section{Flexor-pronator tendinosis or rupture}

Unlike to the common "tennis elbow", or lateral epicondylitis, this tendinosis is more common in high-level tennis players than it is in recreational players. The pronator teres and flexor carpi radialis have been identified as the most common sites of pathologic changes. ${ }^{17} 18$ Athletes complain about tenderness distal and lateral to the medial epicondyle; resisted wrist flexion and forearm pronation exacerbate pain.

Treatment is in general a non-operative program for at least 6 months; persistent symptoms after 6 months can be an indication for surgical treatment after exclusion of any other pathologic causes, especially UCL insufficiency. Medial epicondylitis represents an "absolute overload" of normal anatomy and physiology due to supra normal forces; possible related factors are an excessive wrist snap, "open stance hitting", opening too soon on serve and short arming of the strokes. ${ }^{5}$ More research needs to be performed to clarify the relation of biomechanics in tennis and flexor-pronator tendinosis.

\section{Ulnar neuritis}

The cause of ulnar neuritis in the cubital tunnel is considered to be the result of mechanical stimuli on the ulnar nerve in the cubital tunnel.

Ulnar neuritis around the elbow can be the result of compression or traction from valgus stress and can be seen as an isolated injury or in combination with UCL insufficiency or chronic flexor pronator mass tendinosis. Compression can occur due to a tight cubital tunnel, osteophytes from the ulnohumeral joint, muscle hypertrophy or subluxation of the nerve. In tennis players, the initial presentation of ulnar neuritis can be pain along the medial joint line associated with dysesthesias, paresthesias or even anaesthesia in the small and ulnar half of the ring finger. The degree of sensory and motor changes can vary depending on the severity and duration of ulnar nerve compression. Surgical intervention is indicated in case of progressive muscle weakness, persistent muscle weakness for more than 4 months, chronic neuropathy or failure of a nonsurgical regime. ${ }^{19}{ }^{20}$ In a cadaver study, the movement of the ulnar nerve at the proximal aspect of the cubital tunnel was significantly increased during all throwing phases with increased elbow flexion $(\mathrm{p}<0.05)$. A mean $(\mathrm{SD})$ maximum movement of 12.4 (2.4) $\mathrm{mm}$ was recorded during the wind-up phase with maximum elbow flexion. The maximum strain on the ulnar nerve during the acceleration phase was found to be close to the elastic and circulatory limits of the nerve. ${ }^{21}$ Although in this study the "throwing motion" of the elbow was studied, the same principles can probably be applied to motion of the elbow during service. The ulnar nerve is subjected to longitudinal strain in the cubital tunnel during the service motion and this longitudinal strain is increased as the elbow is in greater flexion.

During rehabilitation of ulnar neuritis the amount of flexion during service should be taken into account. As ulnar neuritis can be the result of valgus instability or insufficiency of the UCL the same principles should be applied as described under UCL insufficiency.

\section{Posterior impingement}

Posterior impingement of the elbow is an uncommon disorder in the general population; it is mainly seen in patients that overuse their elbow during specific sporting activities as such overhead throwing or tennis. ${ }^{22}{ }^{23}$ The lesion is due to repetitive combined hyperextension, valgus and suspiration of the elbow resulting in a mechanical abutment of bony or soft tissues in the posterior fossa of the elbow. Posterior impingement can also be associated with ligamentous instability of the elbow, especially UCL insufficiency. In a cadaver study, valgus torques of 1.25 and $2.0 \mathrm{Nm}$ were applied and kinematic data were obtained with intact and transected UCL at different angles of flexion angle using a 3-dimensional digitiser. For a given load and flexion angle, the contact area decreased and the pressure increased with increasing medial ulnar collateral ligament insufficiency. The conclusion was that medial ulnar collateral ligament insufficiency alters contact area and pressure between the posteromedial trochlea and olecranon and helps explain the development of posteromedial osteophytes in cases of UCL insufficiency. ${ }^{24}$

The athlete complains of pain posteriorly, joint effusion, locking, crepitus and a decrease in range of motion, most notably an extension deficit. If conservative treatment of 
posterior impingement is not successful, arthroscopic debridement of the elbow with removal of osteophytes and synovectomy can be used in these patients.

In the treatment of posterior impingement, hyperextension of the elbow joint and insufficiency of UCL must be addressed.

\section{Osteochondritis dissecans}

Osteochondritis dissecans (OD) of the elbow is an uncommon disorder in the general population. It is usually seen in patients that overuse their elbow during specific sporting activities in which the elbow is extended forcefully or axially loaded. ${ }^{25}$ It has been described in players with insufficiency of the UCL or players with muscle weakness or hyperlaxity of the elbow joints.

This injury occurs usually in the lateral compartment as a result of shear and compression forces to the particular cartilage or underlying bone of the lateral compartment.

These compressive forces on the radio-humeral joint can become as high as $500 \mathrm{~N}$, resulting in (Osseo) chondral fractures and secondary defects in the radio humeral joint. ${ }^{26}$ Tennis players mostly complain on a dull and aching pain in and around the elbow shortly after demanding activities. Findings during physical examination as swelling, tenderness over the radiohumeral joint and limitations in motion; especially loss of extension is either seen.

Treatment is dependent of the severity, size and location of the lesion and age of onset and can be conservative or surgical. Underlying valgus instability should be addressed accordingly and hyperextension should be avoided.

\section{Lateral epicondylitis}

Lateral epicondylitis is 7-20 times more common than its medial counterpart and produces pain along the lateral elbow and forearm. Treatment is generally conservative. In cases lasting more than a year, surgery can be considered.

Lateral humeral epicondylitis is a condition that primarily occurs in the recreational tennis player. One of the reasons is an increase of wrist extension in more experienced players just prior to ball impact. Novice players strike the ball with their wrist in more flexed position at impact. ${ }^{27}$

Observations of the patterns of activation and joint kinematics of novice tennis and advanced payers, using kinematic data in conjunction with a computer model, have revealed substantial eccentric contractions of the extensor carpi, which are likely the cause of repetitive microtrauma leading to tennis elbow injuries. Adopting the technique seen in advanced players probably helps limit the eccentric contractions and reduces the likelihood of injury. ${ }^{28}$ Tennis grip size was believed to play a crucial role in the past. However, based on fine-wire electromyography studies in which muscle activity in extensor carpi radialis longus and brevis, extensor digitorum communis, flexor carpi radialis and pronator teres were measured, tennis racquet grip size (1/4) above or below Nirschl's recommended measurement does not significantly affect forearm muscle firing patterns. ${ }^{29}$ Alterations in tennis racquet grip size do not have a significant effect on forearm muscle activity and therefore might not represent a significant risk factor for lateral epicondylitis.

The unusual EMG findings of increased activity in injured muscles can be explained by faulty mechanics that predispose to the development of tennis elbow. It is therefore clear that concentric and eccentric training should be performed for the forearm muscle, as muscle imbalances will lead to injury in lateral epicondylitis.

\section{CONCLUSIONS}

Tennis places the ligamentous, osseous, musculotendinous and neural structures of the elbow at increased risk for various

\section{What is already known on this topic}

- The biomechanics of tennis have been well documented.

- The clinical symptoms of tennis-related injuries and their treatment have been published over a wide range of journals.

\section{What this study adds}

The most important biomechanical aspects of tennis in relation to the treatment of the tennis-related injuries are described.

injuries. Proper training and preventive exercise, based on sound biomechanical research, can result in decrease of loads across the elbow in tennis players. It is important to recognise that injuries can occur simultaneously and that every entity must be treated accordingly.

The kinetic chain of the tennis service and strokes should be taken into account. For example tennis players with more effective knee flexion-extension during the service action were associated with lower loading at the shoulder and elbow. ${ }^{30}$ The exact impact of this finding is as yet unknown and needs to be further investigated. ${ }^{5}$

Internal rotation of the upper arm at the shoulder during the service and forehand is of utmost importance; decrease of internal rotation in the shoulder can increase rotatory stress on the elbow. Internal rotation of the upper arm and pronation of the forearm during the early phase of the follow-through of the service probably reduce these forces on the elbow. ${ }^{2}$ Grip size does not seem to play a role in elbow injuries; playing with a "Western grip" can possibly increase valgus stress on the elbow, especially during acceleration. ${ }^{2}$

Strengthening the forearm flexor muscles and reducing elbow extension after impact might help reduce injury risk.

In general, most symptomatic conditions of the elbow in tennis players can be treated conservatively initially. In cases where conservative treatment is not successful, surgical intervention is indicated. In conservative and surgical treatment protocol analysis of the biomechanics of each tennis player must be performed and abnormalities addressed.

\section{Authors' affiliations \\ Denise Eygendaal, Amphia Hospital, Teteringen, The Netherlands F Th G Rahussen, Sint Maartenskliniek, Nijmegen, The Netherlands R L Diercks, UMCG, Gronigen, The Netherlands}

Competing interests: None declared.

\section{REFERENCES}

1 Letsel Informatie Systeem 1998-2001. Ongevallen tijden racketsporten. Amsterdam: Consument en Veiligheid, 2003.

2 Pluim B, Safran M. From breakpoint to advantage. Vista, California, USA: Racquet Tech Publishing, 2004.

3 Morrey BF, Tanaka S, An KN. Valgus stability of the elbow: a definition of primary and secondary constraints. Clin Orthop Rel Res, 1991:265;187-95.

4 Regan WD, Korinek S, Morrey BF, An KN. Biomechanical study of ligaments around the elbow joint. Clin Orthop Rel Res 1991;271:170-9.

5 Kibler WB. Clinical biomechanics of the elbow in tennis: implications for evaluation and diagnosis. Med Sci Sports Exerc 1994;26:1203-6.

6 Elliott B. Biomechanics and tennis. Br J Sports Med 2006;40:392-6.

7 Fleisig GS, Andrews JR, Dillman CJ, et al. Kinetics of baseball pitching with implications about injury mechanisms. Am J Sports Med 1995;23:233-9.

8 Andrews JR, Whiteside JA. Common elbow problems in the athlete. J Orthop Sports Trauma 1993;17:289-95.

9 Conway JE, Jobe FW, Glousman RE, et al. Medial instability of the elbow in throwing athletes. Am J Bone Joint Surg 1992;74:67-83. 
10 Indelicato PA, Jobe FW, Kerlan RK, et al. Correctable elbow lesions in professional baseball players: a review of 25 cases. Am J Sports Med 1979:7:72-6.

11 Jobe FW, Stark H, Lombardo SJ. Reconstruction of the ulnar collateral ligament in athletes. Am J Bone Joint Surg 1986;68:1158-63.

12 Safran MR. Elbow injuries in athletes. A review. Clin Orthop Relat Res 1995; 310:257-77.

13 Eygendaal D, Safran MR. Postero-medial elbow problems in the adult athlete. Br J Sports Med 2006;40:430-4.

14 Andrews JR. Bony injuries about the elbow in the throwing athlete. Instr Course Lect 1985:34:323-331.

15 Breazeale NM, Dines JM, Altchek DW. Chondromalacia of the humeral troclea in throwing athletes: description of a new chondral lesion with biomechanical correlation. Paper presented at American Orthopaedic Society for Sports Medicine, Vancouver, 1999

16 Davidson PA, Pink M, Perry J, et al. Functional anatomy of flexor pronator muscle group in relation to the MCL of the elbow. Am J Sports Med 1995:23:245-50.

17 Nirschl RP, Pettrone FA. Tennis elbow: the surgical treatment of lateral epicondylitis. J Bone Joint Surg Am 1979;61:832-9.

18 Vangsess CT Jr, Jobe FW. Surgical treatment of medial epicondylitis. Results in 30 elbows. J Bone Joint Surg 1991;73:409-11.

19 Eisen A, Danon J. The mild cubital tunnel syndrome. Its natural history and indication for surgical intervention. Neurology 1974;24:608-13.

20 Posner MA. Compressive ulnar neuropathies at the elbow: II. Treatment. J Am Acad Orthop Surg 1998;6:289-97.
21 Aoki $M$, et al. Strain on the ulnar nerve at the elbow and wrist during throwing motion. J Bone Joint Surg Am 2005;87:2508-14.

22 Fideler BM, Kvitne RS, Jordan S. Posterior impingement of the elbow in professional baseball players. J Shoulder Elbow Surg 1997;6:169-70.

23 Bradley JP. Arthroscopic treatment of posterior impingement of the elbow in NFL lineman. J Shoulder Elbow Surg 1995;2:119-20.

24 Ahmad CS, Park MC, Elattrache NS. Elbow medial ulnar collateral ligament insufficiency alters posteromedial olecranon contact. Am J Sports Med 2004;32:1607-12.

25 Stubbs MJ, Field LD, Savoie FH 3rd. Osteochondritis dissecans of the elbow. Clin Sports Med 2001;20:1-9.

26 Sato M, Ochi M, Uchio Y, et al. Transplantation of tissue-engineered cartilage for excessive osteochondritis dissecans of the elbow. J Shoulder Elbow Surg 2004; 13:221-5.

27 Blackwell J, Cole K. Wrist kinematics differ in expert and novice tennis players performing the backhand stroke. Implications for tennis elbow. J Biomech 1994:27:509-16.

28 Riek S, Chapman AE, Milner T. A simulation of muscle force and internal kinematcis of extensor carpi radialis brevis during backhand tennis stroke; implications for injury. Clin Biomech 1999;14:477-83.

29 Hatch GF 3rd, Pink MM, Mohr KJ, et al. The effect of tennis racket grip size on forearm muscle firing patterns. Am J Sports Med 2006;34:177-83.

30 Elliott B, Fleisig G, Nicholls R. Technique effects on upper limb loading in the tennis serve. J Sci Med Sport 2004;6:76-87. 